\title{
The spectral energy distribution of self-gravitating protostellar disks
}

\author{
G. Lodato ${ }^{1}$ and G. Bertin ${ }^{1,2}$ \\ 1 Scuola Normale Superiore, Piazza dei Cavalieri 7, 56126 Pisa, Italy \\ 2 Università degli Studi di Milano, Dipartimento di Fisica, Via Celoria 16, 20133 Milano, Italy
}

Received 12 February 2001 / Accepted 12 June 2001

\begin{abstract}
The long wavelength emission of protostellar objects is commonly attributed to a disk of gas and dust around the central protostar. In the first stages of disk accretion or in the case of high mass protostars, the disk mass is likely to be sufficiently large, so that the disk self-gravity may have an impact on the dynamics and the emission properties of the disk. In this paper we describe the spectral energy distribution (SED) produced by a simple, non-flaring, self-gravitating accretion disk model. Self-gravity is included in the calculation of the rotation curve of the disk and in the energy balance equation, as a term of effective heating related to Jeans instability. In order to quantify in detail the requirements on the mass of the disk and on the accretion rate posed on the models by realistic situations, we compare the SEDs produced by these models with the observed SEDs of a small sample of well-studied protostellar objects. We find that relatively modest disks - even lighter than the central star - can lead to an interesting fit to the infrared SED of the FU Orionis objects considered, while in the case of $\mathrm{T}$ Tauri stars the required parameters fall outside the range suggested as acceptable by the general theoretical and observational scenario. On the basis of the present results, we may conclude that the contribution of a selfgravitating disk is plausible in several cases (in particular, for FU Orionis objects) and that, in the standard irradiation, dominated disk scenario, it would help soften the requirements encountered by Keplerian accretion models.
\end{abstract}

Key words. accretion, accretion disks - gravitation - stars: pre-main sequence

\section{Introduction}

It now seems well established that pre-main-sequence stars, such as T Tauri and FU Orionis objects, are generally surrounded by a disk of gas and dust. The traditional evidence for such circumstellar disks comes from the excess luminosity found at long wavelengths, far-infrared (Rydgren et al. 1976) and millimetric (Beckwith et al. 1990), indicating that this radiation comes from a region of relatively low temperature, far from the central star.

The first attempt to try explain such excess infrared luminosity dates back to the 70s (Lynden-Bell \& Pringle 1974), when it was argued that it might be due to the release of gravitational binding energy from an accretion disk. In recent years, the general picture that has emerged is that, in most $\mathrm{T}$ Tauri systems, the mass accretion rate is too low $\left(\dot{M} \sim 10^{-8}-10^{-7} M_{\odot} /\right.$ yr $)$ to account for the observed luminosity and that the observed long wavelength excess is likely to result from the reprocessing of the starlight from the outer disk (Hartmann 1998). Therefore, the accretion disk of $\mathrm{T}$ Tauri stars is generally thought to be

Send offprint requests to: G. Lodato,

e-mail: lodato@cibs.sns.it "passive". In contrast, during FU Orionis outbursts, the accretion rate appears to be relatively high, so that here the disk can dissipate sufficient amounts of energy to produce the observed luminosity. In the construction of dynamical models, a related issue is that of the total disk mass. Clues have indeed been gathered that the disk mass may be large in some protostellar systems (see Lay et al. 1994), especially in the earlier phases of the process of star formation (Yorke \& Bodenheimer 1999, for example, conclude from hydrodynamical simulations that the disk mass at the beginning of the accretion phase is comparable to that of the central object) and in the context of high-mass star formation, where observations point to the presence of massive disks (Cesaroni et al. 1994).

Standard accretion disk theory (Shakura \& Sunyaev 1973) is based on the assumption that the disk is optically thick and geometrically thin and that the power viscously dissipated in the disk $D=\nu_{\alpha} \sigma\left(r \Omega^{\prime}\right)^{2} / 2$ is all radiated away. In the optically thick case, it can be shown that the spectral index $n=\mathrm{d} \log \left(\nu F_{\nu}\right) / \mathrm{d} \log \nu$ in the infrared part of the spectrum is related to the exponent $q$ in the surface temperature profile $T_{\mathrm{s}}=T_{0}\left(r_{0} / r\right)^{q}$, so that $n \simeq 4-2 / q$ (Adams et al. 1988). For a Keplerian rotation curve one 
has $T_{\mathrm{S}}(r) \propto r^{-3 / 4}$, leading to $L_{\nu}=\nu F_{\nu} \propto \nu^{4 / 3}$, while for $\mathrm{T}$ Tauri stars one typically finds $L_{\nu} \propto \nu^{2 / 3}$ (Kenyon \& Hartmann 1995). The difficulty becomes even more evident for the so-called "flat-spectrum T Tauri" (such as T Tau itself and HL Tau), which show a spectral index $n \approx 0$. In turn, if the rotation curve is flat, the surface temperature profile decreases more slowly, as $T_{\mathrm{S}}(r) \propto r^{-1 / 2}$. In this latter case, the spectral index would be $n=0$, resembling that of flat-spectrum objects. Adams et al. (1988) indeed proposed that the flat spectrum could arise from a disk with such a non-standard surface temperature profile, but argued that the observed disk mass in these systems (although difficult to measure) is probably too low to produce substantial changes in the rotation curve and then suggested that the non-standard temperature profile should be ascribed to collective processes of angular momentum and energy transport associated with self-gravity-induced instabilities.

In the absence of a convincing physical justification for non-standard descriptions of accretion disks, the attention has been turned to other ways of explaining the far-infrared spectra of T Tauri stars. As noted above, it is now commonly thought that in most of these systems the accretion rate is low and that the heating of the disk is primarily due to irradiation from the central star. The effect of irradiation is enhanced at large radii by the disk flaring, when the thickness of the disk increases rapidly with radius. Models of the vertical structure of these flared disks have been constructed (Kenyon \& Hartmann 1987; Chiang \& Goldreich 1997; but concerns about stability have been expressed by Dullemond 2000). On the other hand, in the case of flat spectrum sources and of most FU Orionis objects, flaring alone is ineffective and the far-infrared emission has been attributed to an infalling envelope of dust (Kenyon \& Hartmann 1991; Calvet et al. 1994).

The hypothesis that the flat long wavelength spectrum of protostellar objects arises from a self-gravitating disk with flat rotation curve actually remains attractive from a physical point of view, especially for its simplicity. Unfortunately, only scattered efforts have been made in the direction of constructing self-gravitating models of accretion disks and little or no physical justification has been provided for the desired radial surface density profile. The general ideas mentioned above have been revisited recently along with a new viscosity prescription for self-gravitating disks (Duschl et al. 2000). In view of this discussion, it seems natural to consider and to put to a test the accretion disk models that we have constructed recently (Bertin 1997; Bertin \& Lodato 1999, hereafter BL99), which include the gravitational fields of both the central star and the disk. Interestingly, these models show that the rotation curve of a self-regulated accretion disk is Keplerian in the inner disk and flat in the outer disk, corresponding to a surface density $\sigma \propto r^{-1}$ (which, incidentally, has been observed in some cases: e.g., TW Hya, Wilner et al. 2000, and HH 30; Burrows et al. 1996), the transition taking place at a radius $r_{\mathrm{s}} \approx\left(G M_{\star} / 8\right)(G \dot{M} / 2 \alpha)^{-2 / 3}$ (using standard notation; see Sect. 2). Deviations from Keplerian rotation occur even for disk masses $M_{\text {disk }} \lesssim M_{\star}$, which could be a plausible range even for some $\mathrm{T}$ Tauri disks. Based on these models of self-regulated accretion, it is now possible to quantify in detail what would be the parameter requirements needed to fit the infrared spectra of protostellar objects. In this respect, our results will turn out to be quite different (see discussion in Sect. 3.5) from earlier estimates (e.g., see Kenyon \& Hartmann 1987; Shu et al. 1987) that did not include the important ingredient of self-regulation, which is likely to change significantly the energy balance equations in the self-gravitating part of the disk (see also discussion in Sect. 2.2).

In this paper we describe the spectral energy distribution produced by a class of self-regulated, self-gravitating accretion disks and discuss its dependence on the various parameters involved and the differences from the non-selfgravitating case. In addition, we determine the physical parameters needed to fit the SEDs of realistic protostellar objects, by referring to a small sample of well-studied FU Orionis systems and T Tauri stars. A general theory of FU Orionis and T Tauri systems, or a comprehensive picture of the individual objects picked here to test the pure self-gravitating model against observed SEDs, is beyond the goals of this paper. The models considered are extremely simple and do not address the issue of the physical ingredients that determine the long-term evolution of the system (in particular, the physical ingredients responsible for the outburst phase of the FU Orionis systems): they are taken to match current "snapshots" of such evolving systems, assuming that the steady-state equations are temporarily adequate to describe the observed situation.

The quality of the fit to the available data for the long wavelength spectral energy distribution is very good. In the case of FU Orionis systems the required accretion rate is of the order of $\dot{M} \approx 10^{-5}-10^{-4} M_{\odot} / \mathrm{yr}$ and the implied disk mass is of the order of one solar mass. The implied mass accretion rate for $\mathrm{T}$ Tauri stars is found to be $\dot{M} \approx 10^{-7}-10^{-6} M_{\odot} /$ yr. The choice of the outer radius of the disk determines the mass of the disk relative to that of the central star. The estimates, although uncertain, point to rather massive disks $\left(M_{\text {disk }} \approx 0.3 M_{\odot}\right)$. In the case of FU Orionis systems the mass accretion rates are in reasonable agreement with the currently accepted estimates, while the disk masses are higher than the commonly assumed disk masses in these systems. For T Tauri stars, instead, the required parameters tend to fall outside the range suggested as acceptable by the general theoretical and observational scenario. In any case, the contribution of self-gravity may soften the demands on models that attempt a description without the use of the disk self-gravity.

The paper is organized as follows. In Sect. 2 we describe the model of self-gravitating, self-regulated accretion disk that we adopt here; in Sect. 3 we describe the spectral energy distributions derived on the basis of our model and discuss their general properties; in Sect. 4 we consider a sample of FU Orionis objects and fit their SED with the self-gravitating disk model, deriving the physical 
properties of the disks inferred on the basis of our model, and discuss the results obtained; in Sect. 5 we point out the difficulties met when trying to adopt a similar approach for the case of $\mathrm{T}$ Tauri stars; in Sect. 6 we draw our conclusions.

\section{Self-regulated accretion disks}

\subsection{Disk model}

We refer to a self-gravitating accretion disk model that is only partially self-regulated (BL99), so that the relevant $Q$-profile is of the form:

$Q=\frac{c_{\mathrm{s}} \kappa}{\pi G \sigma}=\bar{Q}\left[1+\left(\frac{r}{r_{Q}}\right)^{-9 / 8} \exp \left(-r / r_{Q}\right)\right]$,

where $c_{\mathrm{s}}$ is the effective thermal speed, $\kappa$ is the epicyclic frequency, and $\sigma$ is the surface density of the disk. In this way the stability parameter $Q$ is much higher than unity in the inner disk $\left(r \lesssim r_{Q}\right)$ and becomes constant $(Q \sim \bar{Q} \approx 1)$ in the outer disk. While in the context of galactic dynamics the scale $r_{Q}$ is usually associated with the bulge size and, in the context of AGN, some efforts have been made in the description of the transition from a non-self-gravitating to a self-gravitating accretion disk (Bardou et al. 1998), no clear-cut prescription for the onset of self-regulation in protostellar disks is available. We have thus decided to take $x_{Q}=r_{Q} / r_{\mathrm{s}}$ (for the definition of $r_{\mathrm{s}}$, see Eq. (2) below) as a free parameter of our model. The scale $r_{\mathrm{s}}$ is the scale that defines the transition from Keplerian to flat rotation curve:

$r_{\mathrm{s}}=2 G M_{\star}\left(\frac{\bar{Q}}{4}\right)^{2}\left(\frac{G \dot{M}}{2 \alpha}\right)^{-2 / 3}$.

Here $M_{\star}$ is the mass of the central object, $\dot{M}$ the accretion rate, and $\alpha$ the viscosity parameter of the Shakura \& Sunyaev (1973) prescription

$\nu_{\alpha} \sigma=\alpha c_{\mathrm{s}} h \sigma=\frac{\alpha c_{\mathrm{s}}^{3}}{\pi G} \tilde{h}(Q, \Omega / \kappa) ;$

in the last equation we calculate the disk thickness as $h=$ $\left(c_{\mathrm{s}}^{2} / \pi G \sigma\right) \tilde{h}$, with:

$\tilde{h}=\frac{\pi}{4 Q^{2}\left[2 \Omega^{2} / \kappa^{2}-1\right]}\left[\sqrt{1+\frac{8}{\pi} Q^{2}\left(\frac{2 \Omega^{2}}{\kappa^{2}}-1\right)}-1\right]$.

The last expression gives a useful interpolation formula for the thickness of the disk in the case when the contributions of the star and of the disk are both taken into account in the vertical gravitational field (see BL99, Appendix).

In the following, for simplicity we assume that the conservation law for the angular momentum is written as:

$G \dot{M} r^{2} \Omega+2 \alpha c_{\mathrm{s}}^{3} \tilde{h} r^{3} \frac{\mathrm{d} \Omega}{\mathrm{d} r} \simeq 0$

and thus avoid the relatively complex behavior that is associated with a net transport of angular momentum
$(\dot{J} \neq 0$; calculated by BL99). Here we may also recall that Popham et al. (1996) find better agreement with the observational data when their boundary layer models for FU Orionis systems are taken with a negligible value of $\dot{J}$.

Finally, the rotation curve is computed by solving the full Poisson equation, including the contribution of both the central star and the disk:

$V^{2}=\frac{G M_{\star}}{r}+r \frac{\mathrm{d} \Phi_{\sigma}}{\mathrm{d} r}$,

where the gravitational field of the disk $\mathrm{d} \Phi_{\sigma} / \mathrm{d} r$ is given in Eq. (4) of BL99. In practice, a useful way to express the rotation curve is by scaling $V^{2}$ to its value in the outer disk:

$V^{2}=\frac{1}{2}\left(\frac{4}{\bar{Q}}\right)^{2}\left(\frac{G \dot{M}}{2 \alpha}\right)^{2 / 3} \phi^{2}$,

with $\phi^{2}$ given in Eq. (13) of BL99.

Equation (5) gives an expression for the profile $c_{\mathrm{S}}(r)$ in terms of $\Omega(r)$ and $Q(r)$; the latter functions are available from Eqs. (7) and (1). Similarly, Eq. (3) provides the profile of the quantity $\nu_{\alpha} \sigma$. Therefore, we have an explicit expression, dependent on few parameters (such as $\alpha, \dot{M}$, $\bar{Q})$, for the viscous dissipation rate $D(r)=\nu_{\alpha} \sigma\left(r \Omega^{\prime}\right)^{2} / 2$. In the standard theory this would be sufficient to specify the surface temperature profile $T_{\mathrm{s}}(r)$ associated with the optically thick emission of the disk. Here, as described in a separate article (Bertin \& Lodato 2001, hereafter BL01; see also additional remarks in Sect. 2.2 below), it is appropriate to consider an additional heating term in the energy balance equation, so that the surface temperature profile is determined by:

$\sigma_{\mathrm{B}} T_{\mathrm{s}}^{4}=\frac{1}{2} \nu_{\alpha} \sigma\left(r \Omega^{\prime}\right)^{2}+\frac{g(Q)}{Q} \frac{c_{\mathrm{s}}^{3}}{2 \pi G} \kappa \Omega$,

with $g(Q)=(\bar{Q} / Q)^{m}, m$ a large number (we will take $m=20)$, and where $\sigma_{\mathrm{B}}$ is the Stefan-Boltzmann constant. The additional Jeans-related term ensures that the process of self-regulation properly occurs. Note that in Eq. (8) we neglect the contribution to the heating of the disk associated with the reprocessing of the light emitted by the central star, which is commonly thought to be the key heating source for $\mathrm{T}$ Tauri disks, especially if the outer disk is "flared"; we will discuss the effects of disk irradiation below (Sect. 3.4). In any case, our model of self-gravitating disk has negligible flaring (see BL99).

As a final remark, we note that the assumption that the emission of the disk is optically thick is not conclusive, primarily because of the uncertainties in dust opacity. In this respect, our recent analysis of the energy budget in self-regulated disks (BL01), although it was focused on the different context of AGN, has pointed out that selfgravitating disks may be optically thin. Here, for simplicity and for a direct comparison with other studies, we consider models characterized by optically thick emission.

The notation in the present paper is slightly different with respect to earlier articles (in relation to the use of 
$c_{\mathrm{s}}$ and $\nu_{\alpha}$ ), in order to avoid confusion with the universal notation for some physical quantities (speed of light and frequency).

\subsection{Self-gravity, viscosity, and the energy budget}

Where does the energy of the Jeans-related term in Eq. (8) come from? Why is the heating associated with instabilities not necessarily related to $\dot{M}$ (through $\nu_{\alpha} \sigma$ )? The reader is referred to a separate article (BL01) devoted to introducing and justifying our approach. Here we take this opportunity to add some additional comments that might help answer questions such as those posed above.

Earlier attempts at incorporating the role of collective effects associated with the disk self-gravity generally focused on the problem of angular momentum transport and thus on a modification of the viscosity prescription; these approaches (in particular, see Lin \& Pringle 1987, 1990; Bardou et al. 1998) often realized the need for a mechanism of self-regulation, locally sensitive to the value of $Q$. In turn, numerical simulations (e.g., see Laughlin \& Bodenheimer 1994; Laughlin \& Różyczka 1996) have demonstrated that spiral density waves can indeed transport angular momentum so as to act as an "effective viscosity". In this respect, we agree that gravitational instabilities may have the welcome role, in the outer parts of accretion disks, of contributing significantly to the effective viscosity of the disk, and hence directly to the accretion rate $\dot{M}$.

In the approach that we have followed (starting with Bertin 1997) we have argued that the process of selfregulation, which is expected to maintain the outer disk at values of $Q$ of order unity, should result from a modification of the energy equations, so as to take into account the heating induced by gravitational instabilities. The processes involved are complex (see Sect. 3 in BL99 and Sect. 3 in BL01). We should not expect that all the heating rate should be proportional to the angular momentum transport (as the ideal fluid equations with a viscous term generally imply), especially since axisymmetric instabilities are known also to take place and gravitational heating is observed even in collisionless disks (e.g., see the simulations by Hohl 1971 and Hohl 1973). Therefore, we have thought it appropriate to separate the two issues, of the angular momentum and of the energy transport.

In some extremely simple models (e.g., those considered by $N$-body simulations of collisionless disks that bring out the effects of evolution induced by Jeans-related instabilities) one may argue, for example on the basis of the virial constraint, that if, globally speaking, energy is lost by the system, it must ultimately come from the gravitational potential well. Locally, the statement need not be true, given the facts that some energy may be redistributed across the system through global instabilities (see remarks by Adams et al. 1989) and that gravitational energy has little local meaning. Thus, if we refer to Eq. (8), this local relation does neither contradict nor confirm the requirements of the global energy budget. (Note that, much in line with other descriptions of accretion disks, we do not make explicit use of a horizontal energy transport equation.)

This general message, that the entire system cooperates globally to produce effects that show up differently in different parts of the disk, is often apparent from the results of a variety of numerical simulations (e.g., see Laughlin \& Różyczka 1996; Nelson et al. 2000). In particular, several numerical studies have addressed directly the issue of the energy budget when dynamical instabilities are involved (for some recent studies, see Pickett et al. 1998, 2000; Nelson et al. 1998, 2000).

In addition, the disks that mediate the accretion process are actually not isolated: in fact, for the young stellar objects considered in this paper there is a central star with which the disk connects through a possibly hot and radiatively inefficient inner boundary layer (for FU Orionis objects, see Popham et al. 1996) and the disk is embedded in some environment from which the protostellar cloud has originated. These components, external to our set of model equations, represent a significant energy reservoir.

In conclusion, we are not yet ready to produce an explicit set of closed equations to describe the overall energy budget. In fact, to do so we should not only describe the global processes involved in the energy transport and the physical mechanisms active at the boundaries, but also face in detail the way we visualize an extremely complex system, in which the relevant heating and cooling terms may not be described by a simple one-fluid model (for example, for what concerns possible inelastic collisions between clumps). In this respect, it is interesting to see that in many numerical experiments relatively complex systems are generated, with transient interacting structures (Pickett et al. 2000). On the other hand, the simpler heuristic approach that we have undertaken (in line with the spirit of the Shakura \& Sunyaev prescription) can overcome this difficulty and provide insight into the properties of self-gravitating disks.

While more work is certainly needed to set up a satisfactory global model, for the time being we proceed with this set of model equations that appear to possess most of the necessary ingredients required to describe a truly self-gravitating disk.

\section{General properties of the spectral energy distribution of active, self-gravitating accretion disks}

\subsection{Spectral energy distribution for disk dominated cases}

From Eqs. (3), (5), and (8), it is useful to define a temperature scale $T_{0}$ (with frequency $\nu_{0}=k T_{0} / 2 \pi \hbar$ ) given by:

$T_{0}^{4}=\frac{\dot{M}}{2 \pi \sigma_{\mathrm{B}} \alpha^{2}}\left(\frac{2}{\bar{Q}}\right)^{6}\left(\frac{\dot{M}}{M_{\star}}\right)^{2}$, 
with a dimensionless temperature $\hat{T}=T_{\mathrm{s}} / T_{0}$ and a dimensionless frequency $\hat{\nu}=2 \pi \hbar \nu / k T_{0}=\nu / \nu_{0}$.

For a disk inclined at an angle $\theta$ with respect to the observer's line of sight, at a distance $D_{0}$, the luminosity is provided by integration of the Planck spectrum at the local surface temperature $T_{\mathrm{s}}(r)$ between the inner radius $r_{\text {in }}$ and the outer boundary radius $r_{\text {out }}$ of the disk:

$\nu F_{\nu}=L_{\nu}=\frac{\cos \theta}{D_{0}^{2}} \int_{r_{\text {in }}}^{r_{\text {out }}} \frac{4 \pi \hbar \nu^{4}}{c^{2}} \frac{2 \pi r \mathrm{~d} r}{\mathrm{e}^{2 \pi \hbar \nu / k T_{\mathrm{s}}(r)-1}}$.

Using the dimensionless quantities defined above, the resulting spectrum can be written as:

$4 \pi D_{0}^{2} \nu F_{\nu}=L_{\mathrm{D}} \hat{\nu}^{4} \int_{x_{\mathrm{in}}}^{x_{\text {out }}} \frac{x \mathrm{~d} x}{\mathrm{e}^{\hat{\nu} / \hat{T}(x)}-1}$,

where:

$L_{\mathrm{D}}=\frac{32 \pi^{3} r_{\mathrm{s}}^{2} \hbar \nu_{0}^{4}}{c^{2}} \cos \theta=\frac{240 \cos \theta}{\pi^{4} \bar{Q}^{2}} \dot{M}\left(\frac{G \dot{M}}{2 \alpha}\right)^{2 / 3}$.

Note that the scale $L_{\mathrm{D}}$ of the disk luminosity does not depend on $M_{\star}$.

The spectrum of the disk is then completely defined by two scale parameters $L_{\mathrm{D}}$ and $T_{0}$ and by four dimensionless quantities: the dimensionless inner and outer radii of the disk, $x_{\text {in }}=r_{\text {in }} / r_{\mathrm{s}}$ and $x_{\text {out }}=r_{\text {out }} / r_{\mathrm{s}}$, the viscosity parameter $\alpha$, and the dimensionless radius $x_{Q}$, defining the inner boundary of the self-regulated region (see Eq. (1)). The two geometry independent scale parameters, $L_{\mathrm{D}} / \cos \theta$ and $T_{0}$ can be traced back to the values of $\dot{M}$ and $M_{\star}$.

\subsection{Effects generated by variation of the key parameters}

In this subsection we briefly describe the changes in the SED induced by changing some key parameters. We start from a specific reference model, for which the relevant parameters are listed in Table 1 . For such reference model the scalelength $r_{\mathrm{s}}$ turns out to be $r_{\mathrm{s}} \approx 14 \mathrm{AU}$, so that the scalelength beyond which the disk is self-regulated is $r_{Q}=x_{Q} r_{\mathrm{s}} \approx 5.6 \mathrm{AU}$.

Figure 1 illustrates the effects of varying the outer radius of the disk. As expected, increasing the outer radius leads to a higher infrared excess. For comparison, Fig. 1 also shows a model indicated by "Keplerian". This model is computed with the same physical parameters as for the reference self-gravitating model (i.e., the same $\dot{M}$, the same inner and outer radii, etc.), but as if self-gravity were turned off, i.e. by taking a strictly Keplerian rotation curve and by neglecting the additional term proportional to $g(Q)$ in Eq. (8). The self-gravitating model clearly shows a much flatter SED in the far infrared. In contrast, for $r_{\text {out }}=1 \mathrm{AU}$ the two models (self-gravitating and Keplerian) would produce practically coincident SEDs. This is because for $r_{\text {out }}=1 \mathrm{AU}$ the outer radius of the disk is smaller than $r_{Q}$, so that the disk has not become self-gravitating in this case.
Table 1. Parameters of the reference model.

\begin{tabular}{ccc}
\hline \hline Geometry & & $\cos \theta=1$ \\
Accretion parameters & $\dot{M}=10^{-5} M_{\odot} / \mathrm{yr}$ & $M_{\star}=1 M_{\odot}$ \\
Size of the disk & $r_{\text {in }}=5 R_{\odot}$ & $r_{\text {out }}=10 \mathrm{AU}$ \\
Viscosity and self-regulation & $\alpha=10^{-3}$ & $x_{Q}=0.4$ \\
\hline
\end{tabular}

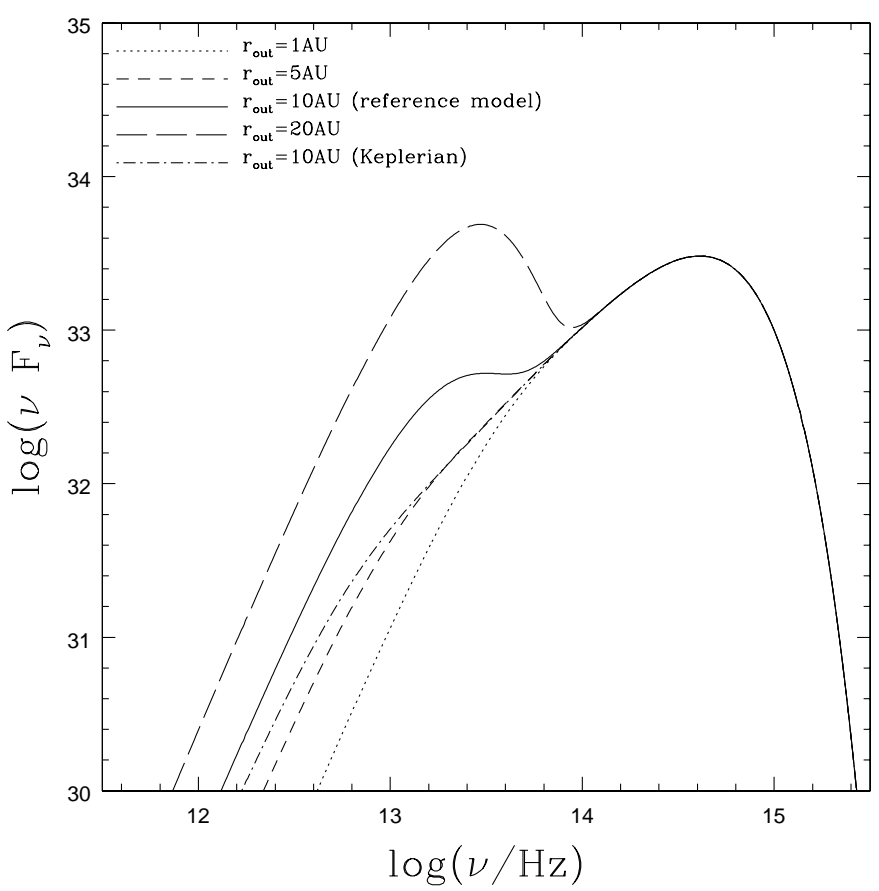

Fig. 1. Shape of the spectral energy distribution for different values of the outer radius $r_{\text {out }}$. The quantity $\nu F_{\nu}$ is in arbitrary units.

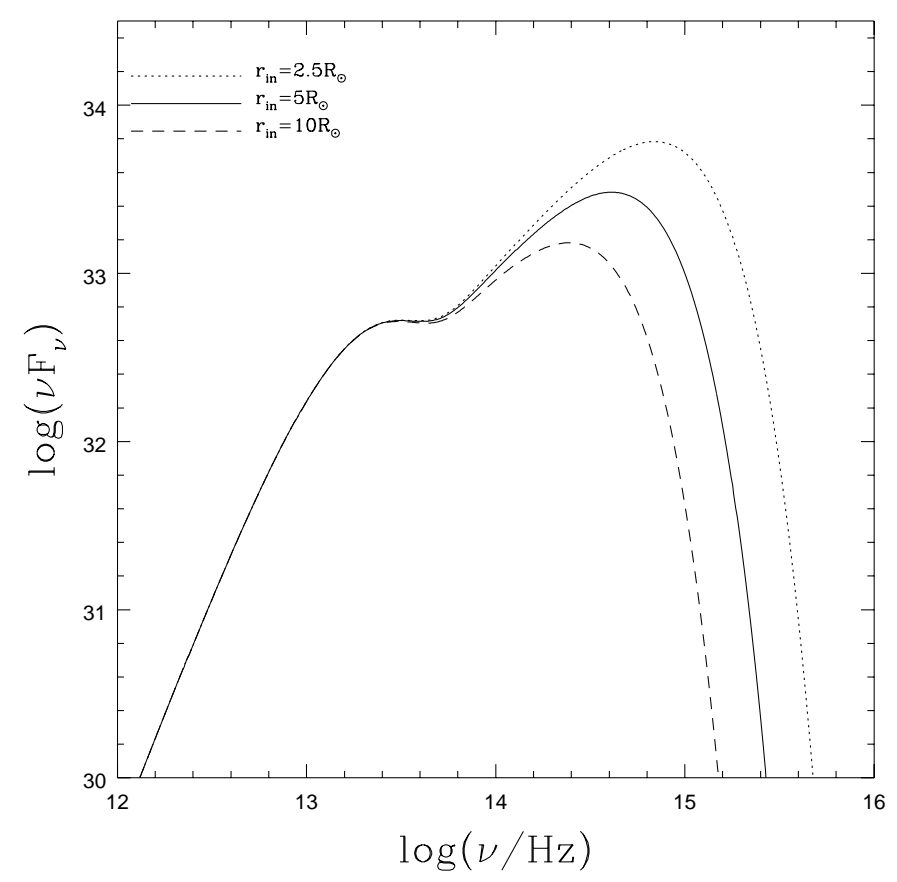

Fig. 2. Shape of the spectral energy distribution for different values of the inner radius $r_{\text {in }}$. The quantity $\nu F_{\nu}$ is in arbitrary units. 


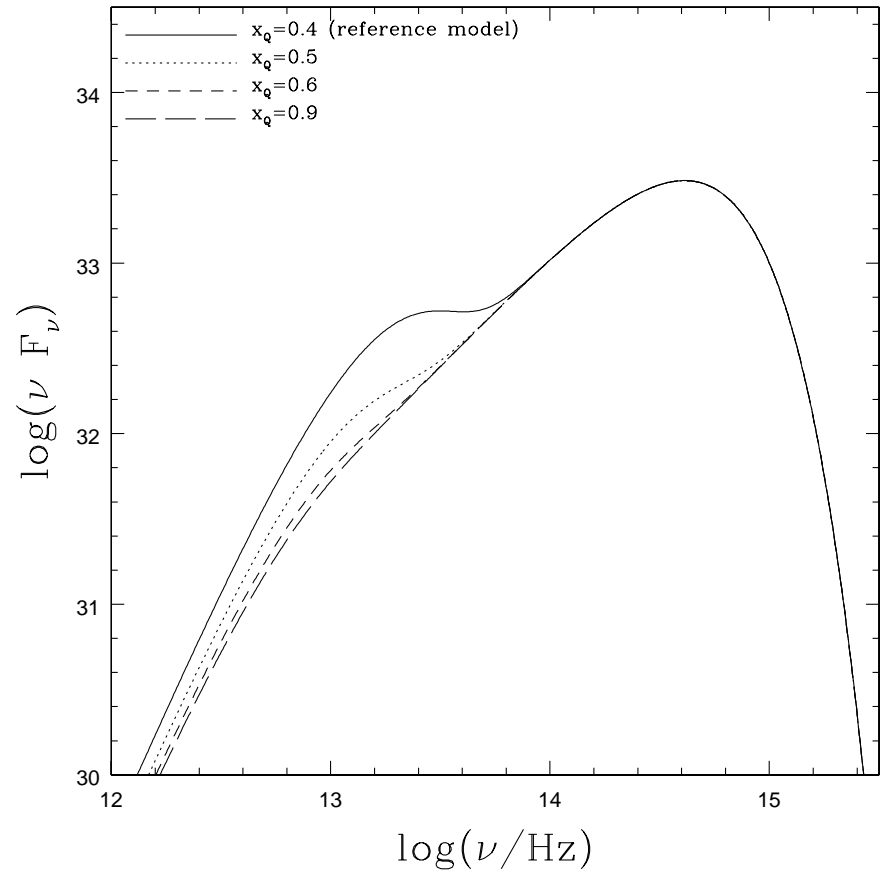

Fig. 3. Shape of the spectral energy distribution for different values of the transition dimensionless radius $x_{Q}$. The quantity $\nu F_{\nu}$ is in arbitrary units.

Figure 2 shows the effects of changing the inner radius of the disk. Not surprisingly, this parameter influences only the optical part of the spectrum. In Fig. 3 we increase the value of $x_{Q}$. For self-gravity to give a substantial contribution to the spectral energy distribution it is necessary that a significant portion of the disk be self-gravitating. For example, for $x_{Q}=0.6$, which corresponds to $r_{Q} \approx 8.5 \mathrm{AU}$ (recall that the outer radius is $\left.r_{\text {out }}=10 \mathrm{AU}\right)$, the SED is still very close to that of the "Keplerian" case.

\subsection{Scaling relations with respect to $\alpha$}

In the previous subsection we have described the changes in the resulting spectral energy distribution in response to changes in the physical parameters of the disk. Here, we will briefly discuss the constraints posed on the relevant parameters, when we consider the problem of fitting the SED of a given object by the self-gravitating accretion disk model.

The highest temperature of the disk, which defines the typical frequency of the optical-near infrared "bump" of the SED, is determined, as in standard accretion disks, by the mass accretion rate, through the relation:

$\sigma_{\mathrm{B}} T_{\mathrm{in}}^{4} \approx \frac{3 G \dot{M} M_{\star}}{8 \pi r_{\mathrm{in}}^{3}}$

independent of the value of $\alpha$. In a similar way, the typical luminosity of the optical-near infrared part of the
SED should be given by the standard non-self-gravitating expression:

$L_{\mathrm{acc}} \approx \frac{G \dot{M} M_{\star}}{2 r_{\mathrm{in}}}$.

Therefore, if the SED of a given object (say, an FU Orionis object) is to be reproduced by a simple disk model, the observed typical wavelength and luminosity of the optical part of the SED (together with an estimate of $M_{\star}$ ), determines the value of $\dot{M}$ and $r_{\text {in }}$ (the latter quantity turns out to be of the order of a few stellar radii; Hartmann \& Kenyon 1996). On the other hand, in the self-gravitating case, the outer disk temperature, which defines the typical frequency of the long wavelength excess, is expected to be associated with a temperature scale $T_{\text {out }}^{4} \approx T_{0}^{4} / \alpha$ (see Eq. (8)). In this way, the simultaneous constraints of fitting the optical and the far infrared parts of the SED define both $\alpha$ and $\dot{M}$.

Conversely, for objects such as T Tauri stars, for which the optical SED is dominated by the central star (see Sect. 3.4 below), the constraints set by Eqs. (13) and (14) do not apply. In turn, under the hypothesis that the far infrared excess is due to the self-gravitating disk (which, however, may not be the case, because irradiation may often dominate), the temperature $T_{\text {out }}^{4} \approx T_{0}^{4} / \alpha$ is still constrained by the observations along with the luminosity scale of the far infrared spectrum, which is set by $L_{\mathrm{D}}$. In this case, when no further information on the accretion rate is given, for a given observed object, from Eqs. (2), (9), and (12) we find the following scaling relations with respect to the viscosity parameter $\alpha$ :

$\dot{M} \propto \alpha$

$r_{\mathrm{s}} \propto(\dot{M} / \alpha)^{-2 / 3}=$ const.

$\cos \theta \propto \dot{M}^{-1}(\dot{M} / \alpha)^{-2 / 3} \propto \alpha^{-1}$.

Indeed, in Section 5 we will see that, for two of the T Tauri objects considered, the exact value of $\alpha$ is not constrained solely by the observed far infrared excess, so that there is room for using different values of $\alpha$. In the other cases shown, the disk gives a contribution also to the higher frequency part of the SED, and so there is little leverage on $\alpha$, as in the disk dominated case.

\subsection{Inclusion of the central star and of disk irradiation}

During FU Orionis outbursts the luminosity of the disk is much higher than that of the central star, so this latter contribution is generally negligible. In contrast, in the case of $\mathrm{T}$ Tauri objects the situation is reversed, so that the stellar luminosity is much higher than that of the accretion disk. There can be however mixed situations in which both contributions should be taken into account. In this case, we should add to the right hand side of Eq. (10) the contribution from the central star, which, for the 
limited purpose of this paper, can be taken to be that of a blackbody with given temperature:

$\nu F_{\nu \star}=\frac{\pi R_{\star}^{2}}{D_{0}^{2}} \frac{4 \pi \hbar \nu^{4}}{c^{2}} \frac{1}{\mathrm{e}^{2 \pi \hbar \nu / k T_{\star}}-1}$.

For convenience, we define a frequency $\nu_{\star}=k T_{\star} / 2 \pi \hbar$. The resulting spectrum in terms of the dimensionless quantities defined in Sect. 3.1 is then given by:

$$
\begin{aligned}
4 \pi D_{0}^{2} \nu F_{\nu}= & \frac{15 L_{\star}}{\pi^{4}}\left(\frac{\nu_{0}}{\nu_{\star}}\right)^{4} \frac{\hat{\nu}^{4}}{\mathrm{e}^{\nu_{0} \hat{\nu} / \nu_{\star}}-1} \\
& +L_{\mathrm{D}} \hat{\nu}^{4} \int_{x_{\text {in }}}^{x_{\text {out }}} \frac{x \mathrm{~d} x}{\mathrm{e}^{\hat{\nu} / \hat{T}(x)}-1},
\end{aligned}
$$

where:

$$
L_{\star}=4 \pi R_{\star}^{2} \sigma_{\mathrm{B}} T_{\star}^{4} .
$$

In addition to its direct contribution, the presence of the star affects the spectral energy distribution also through the reprocessing of the starlight by the disk. For a flat disk, the integrated effect of this contribution can be as high as $L_{\star} / 4$. The effects of disk irradiation can be calculated by following Adams et al. (1988), as an additional contribution to the heat balance equation, so that a term $F_{\mathrm{D}}(r)$ should be added to the right-hand side of Eq. (8), with:

$$
\begin{aligned}
F_{\mathrm{D}}(r) & =\frac{\sigma_{\mathrm{B}} T_{\star}^{4}}{\pi}\left[\arcsin \left(\frac{R_{\star}}{r}\right)-\frac{R_{\star}}{r} \sqrt{1-\left(\frac{R_{\star}}{r}\right)^{2}}\right] \\
& \simeq \frac{2 \sigma_{\mathrm{B}} T_{\star}^{4}}{3 \pi}\left(\frac{R_{\star}}{r}\right)^{3},
\end{aligned}
$$

for a flat disk, and where the approximation is valid for $r \gg R_{\star}$. In terms of the dimensionless quantities defined previously, we find:

$\frac{F_{\mathrm{D}}}{\sigma_{\mathrm{B}} T_{0}^{4}}=\frac{2}{3 \pi}\left(\frac{T_{\star}}{T_{0}}\right)^{4}\left(\frac{R_{\star}}{r_{\mathrm{s}}}\right)^{3} \frac{1}{x^{3}}=\frac{a}{x^{3}}$.

The first term on the right-hand-side of Eq. (8), associated with viscous dissipation, gives a dimensionless contribution to the surface temperature profile $\simeq 1 / x^{3}$, so that the parameter $a$ defined in Eq. (22) measures the relative importance of irradiation versus viscous dissipation. On the other hand, at large radii, when self-regulation becomes important, the leading term that determines the surface temperature is the second, Jeans related term, on the right-hand-side of Eq. (8). It can be easily seen that

$a=\frac{2}{3 \pi}\left(\frac{R_{\star}}{G M_{\star}}\right) \frac{L_{\star}}{\dot{M}}$,

where $R_{\star}, M_{\star}$, and $L_{\star}$ are the stellar radius, mass, and luminosity, respectively. As expected, the relative importance of the stellar irradiation grows with increasing stellar luminosity and decreases with increasing mass accretion rate.

In the following, we will generally neglect this irradiation term. We will check a posteriori the consistency of this assumption in the fitting procedure in the case of $\mathrm{T}$ Tauri stars, by calculating $a$ on the basis of the fitted parameters.

In the case of disk flaring, it has been shown (Ruden \& Pollack 1991) that the effect of irradiation is enhanced as:

$F_{\mathrm{D}}(r)=\frac{2 \sigma_{\mathrm{B}} T_{\star}^{4}}{3 \pi}\left(\frac{R_{\star}}{r}\right)^{3}\left[1+\frac{1}{2}\left(\frac{h}{R_{\star}}\right)\left(\frac{\mathrm{d} \ln h}{\mathrm{~d} \ln r}-1\right)\right]$,

in the limit $h, R_{\star} \ll r$. The term $(\mathrm{d} \ln h / \mathrm{d} \ln r-1)$ is determined from the detailed vertical structure of the disk; its value is equal to $1 / 9$ in the model by Kenyon \& Hartmann (1987) or to $2 / 7$ in the model by Chiang \& Goldreich (1997). In our model (see BL99) at large radii the opening angle $h / r$ does not depend on $r$ and hence there is no flaring.

\subsection{Simple estimates of $\dot{M}$ and $M_{\text {disk }}$}

Here we will briefly discuss the simple arguments often provided (see Kenyon \& Hartmann 1987) to estimate the values of the accretion rate $\dot{M}$ and of the disk mass $M_{\text {disk }}$ directly from the observations of T Tauri stars.

In the case in which viscous dissipation is the only source of heating, the disk surface temperature can be expressed approximately as:

$\sigma_{\mathrm{B}} T_{\mathrm{s}}^{4}(r)=-r \Omega \frac{\dot{M}}{4 \pi} \frac{\mathrm{d} \Omega}{\mathrm{d} r}=\frac{\dot{M} \Omega^{2}}{4 \pi}\left|\frac{\mathrm{d} \ln \Omega}{\mathrm{d} \ln r}\right| \approx \frac{G \dot{M} M_{\mathrm{tot}}(r)}{4 \pi r^{3}}$,

where, in the last expression, $M_{\text {tot }}=M_{\star}+M_{\text {disk }}$. This expression, apart from numerical factors of order unity, is valid both in the Keplerian case (where $M_{\text {tot }} \approx M_{\star}$ and $\Omega^{2} \approx G M_{\star} / r^{3}$ ) and in the disk dominated "flat rotation curve" case, where $M_{\text {disk }}(r) \propto r$ and $\Omega \propto 1 / r$. For a disk with a transition from an inner Keplerian region to an outer self-gravitating region with flat rotation curve, the mass of the disk in its outer part is given by $M_{\text {disk }}(r) / M_{\star} \approx r / r_{\mathrm{s}}$, where $r_{\mathrm{s}}$ is the transition radius (see Eq. (2)).

If we try to follow the argument by Kenyon \& Hartmann (1987), for the case of T Tauri stars, in order for the outer disk to produce the required infrared excess at wavelengths of the order of $25-100 \mu \mathrm{m}$, its temperature should be of the order of $100 \mathrm{~K}$. Therefore, the required product $\dot{M} M_{\text {tot }}\left(r_{\text {out }}\right)$ can be estimated by fixing $T_{\mathrm{s}}\left(r_{\text {out }}\right)=100 \mathrm{~K}$ (with $r_{\text {out }} \approx 20 \mathrm{AU}$; but note that a value of $r_{\text {out }} \approx 10 \mathrm{AU}$ would be more appropriate if we refer to the models described in Sect. 5 below). With this procedure one gets $\dot{M} M_{\text {tot }} /\left(10^{-7} M_{\odot}^{2} \mathrm{yr}^{-1}\right) \approx 10^{3}$. Kenyon \& Hartmann (1987) then noted that both alternatives left by an interpretation in terms of an active disk would be highly implausible: $(i)$ the accretion rate is high, at least in the outer disk, while the disk mass remains very small, thus leading to far too high values of the accretion rates $\left(\dot{M}>10^{-5} M_{\odot} /\right.$ yr), or $(i i)$ the disk mass at $r_{\text {out }}$ is very high, of the order of $1000 M_{\odot}$, while the accretion rate remains small $\left(\dot{M} \lesssim 10^{-7} M_{\odot} / \mathrm{yr}\right)$. In this latter case, to 
Table 2. Stellar parameters assumed for the FU Orionis sample stars.

\begin{tabular}{cccc}
\hline \hline star & $M_{\star} / M_{\odot}$ & $A_{V}$ & $D_{0}$ \\
\hline FU Ori & 1 & 2 & $550 \mathrm{pc}$ \\
V1515 Cyg & 1 & 2.8 & $1000 \mathrm{pc}$ \\
\hline
\end{tabular}

have such a high disk mass, the transition radius to the self-gravitating part of the disk should be very small. In fact, according to Kenyon \& Hartmann (1987) the transition would occur at $r=R_{\star}$.

In reality, in a truly self-gravitating case, viscous dissipation is not the only source of heating (see discussion in Sect. 2.2 and BL01), and so Eq. (25) cannot be used. In addition, when a detailed model of self-gravitating accretion disk of the kind adopted in this paper is considered, one sees that the transition radius is not an independent parameter. In particular, from Eq. (2), when $M_{\star}=0.6 M_{\odot}$ and $\dot{M}=10^{-7} M_{\odot} /$ yr, we see that $r_{\mathrm{s}} \approx 40$ AU, so that the implied disk mass need not to be so high. On the other hand, when self-gravity effects are fully incorporated, the surface temperature is going to be higher than expected from the simple estimates associated with Eq. (25). In fact, from Eq. (8), especially when $\alpha$ is small, it is clear that even with mass accretion rates lower than those inferred from arguments based on Eq. (25) for the "smalldisk-mass" active disk, an active disk could produce the required luminosity.

In Sect. 5 this issue will be discussed further, in view of the parameters determined by fitting concrete examples of spectral energy distributions of $\mathrm{T}$ Tauri stars. Here we would like to anticipate that, even though the results, as already suggested in this subsection, show that the required mass accretion rates need not be as high as estimated earlier, for the majority of $\mathrm{T}$ Tauri stars, the picture of a pure, self-gravitating, active disk remains unsatisfactory.

\section{A possible fit to the spectral energy distribution of some FU Orionis systems}

In this section we will compare the model described in the previous Sections with the observations by fitting the spectral energy distribution of some FU Orionis objects. In these systems there is already evidence that the accretion disk is active, i.e. that most of the emission from these objects is probably due to the disk itself, which outshines the central star. There are already clues that disk selfgravity should play a major role in these cases; in particular, most outburst models predict the existence of massive disks (Bell et al. 1995; Hartmann \& Kenyon 1996).

\subsection{A sample of FU Orionis systems}

Currently, there is a handful of known FU Orionis objects, among which the three best studied systems are FU Ori, V1515 Cyg, and V1057 Cyg. The last object shows a rel-
Table 3. Best-fit parameters for two FU Orionis objects. The values in square brackets refer to different choices of the free parameter $x_{Q}$ ranging in the interval [0.4-0.9].

\begin{tabular}{ccc}
\hline \hline & FU Ori & V1515 Cyg \\
\hline $\log \left(\nu_{0} / H z\right)$ & {$[12.00-12.09]$} & {$[11.94-12.12]$} \\
$\cos \theta$ & {$[0.65-0.72]$} & {$[0.94-0.88]$} \\
$\alpha$ & {$\left[3.4 \times 10^{-2}-1.61 \times 10^{-2}\right]$} & $\left.2.17 \times 10^{-3}-1.09 \times 10^{-3}\right]$ \\
$x_{\text {in }}$ & {$[0.0013-0.0018]$} & {$\left[9 \times 10^{-4}-0.0016\right]$} \\
$x_{\text {out }}$ & {$[1.52-2.51]$} & {$[1.04-1.96]$} \\
\hline
\end{tabular}

Table 4. Derived parameters for the FU Orionis objects. The brackets refer to the same choice of $x_{Q}$ as in Table 3 .

\begin{tabular}{ccc}
\hline \hline & FU Ori & V1515 Cyg \\
\hline$\dot{M} /\left(10^{-5} M_{\odot} / \mathrm{yr}\right)$ & {$[8.2-6.6]$} & {$[1.1-1.2]$} \\
$M_{\text {disk }} / M_{\odot}$ & {$[1.7-2]$} & {$[0.91-1.52]$} \\
$r_{\mathrm{s}} / \mathrm{AU}$ & {$[38-26]$} & {$[23-14]$} \\
$\theta$ & {$\left[49^{\circ}-44^{\circ}\right]$} & {$\left[20^{\circ}-28^{\circ}\right]$} \\
\hline
\end{tabular}

atively rapid decline in $B$ magnitude after the outburst, with a decay timescale of a few years, about ten times faster than FU Ori, and thus special care must be taken when comparing observations made at different epochs. For this reason we have not included V1057 Cyg in our sample of FU Orionis systems. In addition to FU Ori and V1515 Cyg, we have also studied V1735 Cyg, which is an "extreme" case, with a huge infrared excess, so that the infrared luminosity is apparently larger than the optical one. Actually, this object is heavily obscured, so that it is very difficult to derive the extinction correction with confidence and hence to extract for it the intrinsic spectral energy distribution. Therefore, although we initially kept this object in our sample, so as to have an idea of how large the disk mass should be when the infrared excess is so large, we do not record here the results of the fit for this case.

The parameters to be determined by the fit are: the characteristic frequency $\nu_{0}$, the dimensionless inner and outer radii of the disk $x_{\text {in }}$ and $x_{\text {out }}$, the inclination angle $\theta$, and the viscosity parameter $\alpha$. We have derived the bestfit parameters for different choices of $x_{Q}$ taken to vary in the interval $[0.4,0.9]$. To derive the physical parameters of the disk (such as the accretion rate and the disk mass), we have to specify the central star mass (see Table 2). In the case of V1735 Cyg, we have made a fit (not shown in detail here) by assuming $A_{V}=0, M_{\star}=2 M_{\odot}$, on the high side, given the unusual infrared luminosity of the object (see also Sect. 3.1). In any case, in contrast with the models that are generally considered, we do not include here irradiation by the inner on the outer disk (our outer disk has no flaring and hence this contribution is negligible) and the possible contribution of an additional infalling envelope.

The optical and near-infrared luminosities are those collected by Kenyon \& Hartmann (1991). The IRAS fluxes are taken from Weaver \& Jones (1992). First the data 

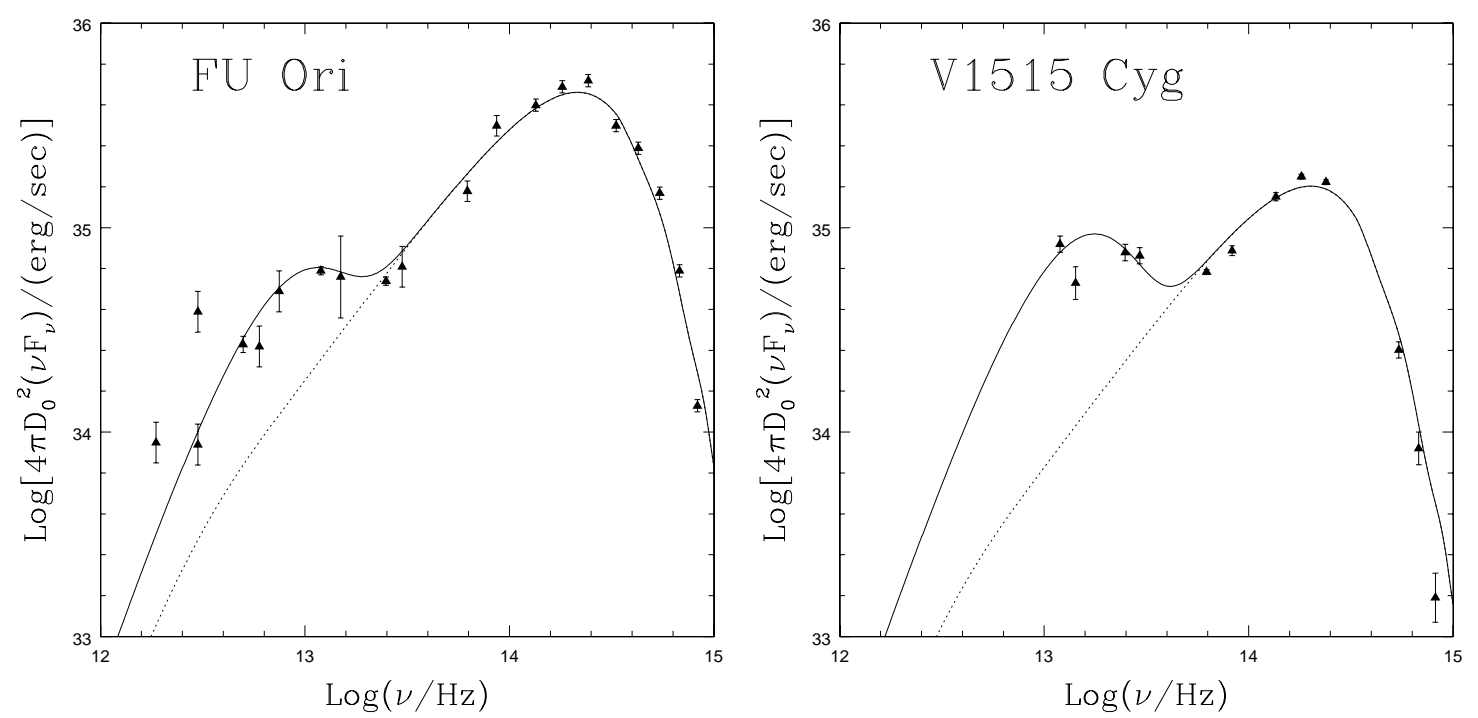

Fig. 4. Best fit to the spectral energy distributions for the FU Orionis sample (solid curves) compared to the spectra resulting from Keplerian disks with the same parameters $\left(\dot{M}, \cos \theta, x_{\mathrm{in}}, x_{\mathrm{out}}, \alpha\right.$; thin curves). Data from Kenyon \& Hartmann (1991) and from Weaver \& Jones (1992).

have been dereddened using the procedure of Cardelli et al. (1989) and then the spectral energy distributions described in the previous section have been fitted to the data.

The best-fit parameters are shown in Table 3 and the derived physical parameters of the disk are shown in Table 4 . Figure 4 displays the spectral data of the stars together with our best-fit SEDs (solid lines) and the corresponding SEDs for non-self-gravitating, Keplerian disks (thin lines), with surface temperature $T_{\mathrm{S}} \propto r^{-3 / 4}$. In Fig. 5 we plot, as an example, the surface temperature profile and the rotation curve for the best-fit model to FU Ori (in the case $x_{Q}=0.4$ ) together with the corresponding quantities for the Keplerian model, showing that the outer parts of the disk in the self-gravitating case are indeed hotter and rotate faster than the corresponding Keplerian disk.

The mass accretion rates are of the order of $10^{-5}-10^{-4} M_{\odot} / \mathrm{yr}$. The value of $\alpha$ determined by the fit is in the range $10^{-3}-10^{-2}$, consistent with some theoretical expectations. Typical disk radii are of the order of 50 AU. Disk masses derived from the fit are of the order of one solar mass. Some of these conclusions would also apply to V1735 Cyg, except that in this case, as a result of its very high infrared excess, the estimated disk mass would be around $20 M_{\odot}$.

\subsection{Discussion}

The infrared spectral energy distribution of FU Orionis systems has been described in terms of active accretion disks by Kenyon et al. (1988) and by Kenyon \& Hartmann (1991). These authors find good agreement between the accretion disk model and the observed spectra for wavelengths smaller than $10 \mu \mathrm{m}$. The excess emission at larger wavelengths is attributed by Kenyon \& Hartmann (1991) to a flattened envelope which reflects the luminosity of the disk. The hypothesis that this excess comes from a flared, outer disk is discarded by Kenyon \& Hartmann (1991), because it would have required unreasonable levels of flaring.

Our estimates of the mass accretion rates are very close to those by Kenyon \& Hartmann (1991), because these numbers are determined mainly by the optical-near infrared part of the spectral energy distribution (see also Sect. 3.3); indeed, as can be seen from Fig. 4, the spectrum for $\lambda<10 \mu \mathrm{m}$ is not affected by self-gravity related effects. On the other hand, in contrast with the interpretation of Kenyon \& Hartmann (1991), we attribute in this paper the far infrared emission to the self-gravitating part of the disk. The mass thus required to produce the observed excess is of the order of one solar mass, which is rather high, but in line with the indications of current outburst models (Bell et al. 1995). Unfortunately, no separate measurements of the disk mass in FU Orionis systems is available to our knowledge.

The interpretation in terms of the presence of an infalling envelope responsible for the far infrared excess is actually appealing because it can also offer a clue to the peculiar time evolution of V1057 Cyg, which shows a correlation between the decay timescale in the optical and that at longer wavelengths $(10 \mu \mathrm{m})$. Here we do not address the time evolution of our model, and indeed we have preferred to leave V1057 Cyg out of our sample. Still, it would be interesting to consider these issues in the context of self-gravitating disks (see also the outburst model of Armitage et al. 2001, which includes some effects related to the disk self-gravity). The far infrared SED in our models is sensitive to the relative positions of the disk outer radius $r_{\text {out }}$ and the radius $r_{Q}$ at which the disk becomes self-regulated (see Sect. 3.2). A detailed description of the role of self-gravity in the context of time-dependent outburst models, which might result from the interplay 

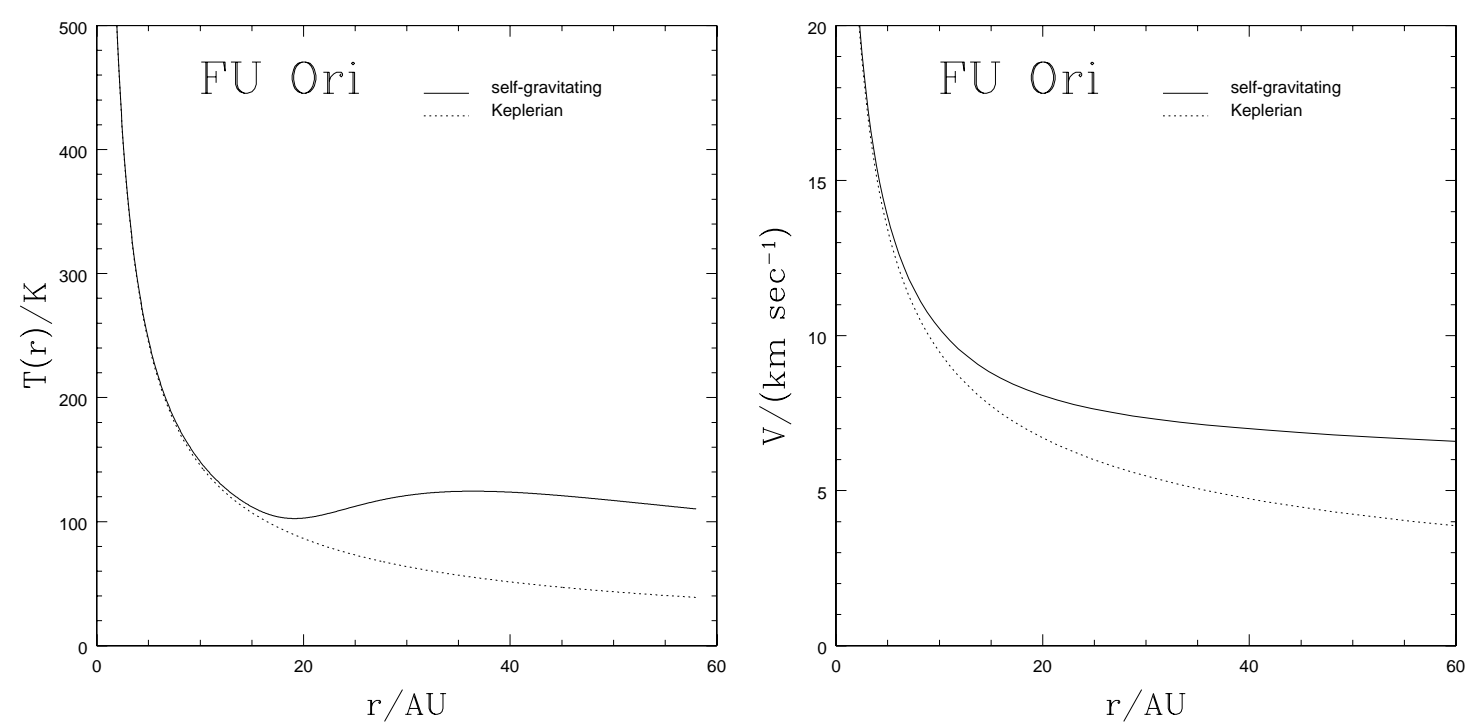

Fig. 5. Surface temperature profile (left) and rotation curve (right) for the best-fit model to FU Ori (with $x_{Q}=0.4$ ), compared with the corresponding Keplerian model.

between $r_{Q}$ and $r_{\text {out }}$ as a function of time, is, however, beyond the goals of this paper.

\section{Difficulties of the self-gravitating scenario in the context of $\mathrm{T}$ Tauri stars}

In the context of $\mathrm{T}$ Tauri stars the situation seems to be less favorable for disk self-gravity to play a major role, because the infrared excess is likely to result in general from irradiation of the disk from the central star. Many points are often mentioned in favor of this interpretation, among which we may recall the explanation of the observed silicate emission features at $10 \mu \mathrm{m}$ (Calvet et al. 1992) and other HST observations (e.g., see Stapelfeldt et al. 1998). On the other hand, initial efforts at explaining the SEDs of $\mathrm{T}$ Tauri stars with active accretion disks led to estimated mass accretion rates so high that the corresponding optical-infrared emission of the disk should outshine the central star, which is not observed. In addition, the peculiar "flat" shape of the far infrared SED, when explained in terms of a non-Keplerian disk, led to unreasonably high disk masses (Kenyon \& Hartmann 1987; Shu et al. 1987).

The model described in this paper presents significant, qualitative and quantitative, differences with respect to the non-Keplerian models considered in previous investigations. First, the additional heating term in Eq. (8) gives an important contribution to the heating of the disk in its self-gravitating part, so that the corresponding mass accretion rates needed to account for the infrared luminosity in an active disk scenario may be lower than previously estimated. Second, as pointed out in Sect. 3.5 and as the fit to FU Orionis objects in the previous section demonstrates, the disk masses needed to give a significant contribution to the SED in the self-gravitating scenario are not as high as previously argued. Therefore, we think it appropriate to reassess the issue of the required parameters within the self-gravitating active disk scenario.
Furthermore, in some cases (see Gullbring et al. 2000; Testi et al. 2001), such as in some intermediate mass Young Stellar Objects, the disk mass may be relatively high, so that the self-gravity of the disk should play some role, even without abandoning the general framework of irradiated disks. In addition, as noted in Sect. 3.5, when $M_{\star} \approx 0.6 M_{\odot}$ and $\dot{M} \approx 10^{-7} M_{\odot} /$ yr (which is not unreasonable at least for some objects), the implied values of $r_{\mathrm{s}}$ readily show that the contribution of disk self-gravity should be incorporated.

For these reasons we now proceed to fit the SEDs of some $\mathrm{T}$ Tauri stars with the model presented in this paper. The primary goal is to check in detail the parameter requirements for the active, self-gravitating disk scenario, so as to confirm or to correct previous estimates, even though this model is going to be relevant only for a small set of less typical cases.

\subsection{A sample of $T$ Tauri stars}

We have considered two typical T Tauri stars (BP Tau and DE Tau) in the Taurus-Auriga molecular cloud (at a distance $D_{0}=140 \mathrm{pc}$ ) for which the stellar masses are available from Gullbring et al. (1998) and the stellar temperatures and visual extinction from Kenyon \& Hartmann (1995). In addition, to check the parameter requirements on our models set by less typical cases, we have considered two "continuum" T Tauri stars, DR Tau and DG Tau, in which the high amount of veiling in the optical suggests the existence of high accretion rates (Gullbring et al. 2000). DG Tau is also one of the "flat spectrum" T Tauri stars considered by Adams et al. (1988). The stellar parameters for the last two cases have been taken from Gullbring et al. (2000), with stellar temperature $T_{\star}=4000 \mathrm{~K}$ and assumed stellar mass of $0.5 \mathrm{M}_{\odot}$.

To fit this set of $\mathrm{T}$ Tauri stars, we add to the disk emission also the direct contribution of the star, as described in 
Sect. 3.4. Nevertheless, we still neglect the effect of irradiation. The consistency of this assumption will be checked a posteriori by estimating the parameter $a$ from the derived fit parameters. This, of course, translates into an actual overestimate of the mass accretion rate, as derived by the fit. The luminosity of the star $L_{\star}$ is added to the set of parameters to be determined by the fit.

The optical and near-infrared luminosities for all stars are available from Kenyon \& Hartmann (1995). The IRAS fluxes for all stars are taken from Weaver \& Jones (1992). The dereddening procedure was the same as adopted for the FU Orionis objects in the previous section. For BP Tau and DE Tau, we found that the value of the viscosity parameter $\alpha$ does not affect significantly the quality of the fit, so it has been excluded from the fit parameters, and the fit has been performed, as separate examples, for two different values: $\alpha=10^{-3}$ and $\alpha=10^{-4}$ (see also comment at the end of Sect. 3.3).

The best-fit parameters (for different values of the parameter $x_{Q}$, taken to vary in the interval $\left.[0.4,0.9]\right)$ are shown in the appendix in Table A.1 and the derived parameters are reported in Table A.2. The fitted SEDs are shown in Fig. 6. As can be seen, the fitted solid curves reproduce the available infrared data well. The surface temperature profile and the rotation curve for DR Tau (in the case where $x_{Q}=0.4$ ) are shown in Fig. 7 . The resulting mass accretion rates for BP Tau and DE Tau are of the order of $10^{-7} M_{\odot} /$ yr for $\alpha=10^{-4}$ and larger by a factor $\approx 10$ for $\alpha=0.001$, as anticipated from the scaling relations pointed out in Sect. 3.3. In these two cases, it can be seen that increasing the value of $x_{Q}$ (with $\alpha$ being fixed) leads to higher disk masses and accretion rates, and consequently to lower values of $r_{\mathrm{s}}$ (which is proportional to $\left.\dot{M}^{-2 / 3}\right)$ and $a\left(\propto \dot{M}^{-1}\right)$. Typical disk radii $r_{\text {out }}$ are of the order of 10-50 AU. The masses required to fit the data are of the order of some fraction of $M_{\odot}$.

For DR Tau we obtain $\dot{M} \approx 5 \times 10^{-7} M_{\odot} /$ yr, while for DG Tau we have $\dot{M} \approx 10^{-6} M_{\odot} /$ yr. The best fit value of $\alpha$ is of the order of $10^{-4}$ and slowly decreases with increasing $x_{Q}$. The required values of the disk masses are a fraction of $M_{\odot}$ also in these cases.

\subsection{Discussion}

For T Tauri stars, the infrared spectral energy distribution is generally not considered to be a good tracer of the mass accretion rate, given the fact that a significant fraction of the infrared luminosity is likely to be due to reprocessing of the starlight rather than to accretion power. Mass accretion rates are better derived from the hot continuum expected to be produced from the accreting matter when it hits the stellar surface (Gullbring et al. 1998). In any case, great uncertainties remain in the derived accretion rates (especially when the hot accretion continuum is very high) mainly due to uncertainties in the extinction corrections. The three most extensive samples of $\mathrm{T}$ Tauri stars with accretion rates determined in this way
(Valenti et al. 1993; Hartigan et al. 1995; Gullbring et al. 1998) report values that differ for the same star by as much as a factor of ten. On the average, the Gullbring et al. (1998) and the Valenti et al. (1993) estimates are consistent with each other, although with large scatter, while the Hartigan et al. (1995) estimates are systematically biased to higher values.

Looking at the values in Table A.2 for the cases of BP Tau and DE Tau, it is clear that the pure selfgravitating disk scenario is unsatisfactory. For $\alpha=10^{-3}$ the values of the required accretion rates for these objects are of the order of $10^{-6} M_{\odot} / \mathrm{yr}$, two orders of magnitude larger than the values typically quoted (Gullbring et al. 1998). This is not surprising, because it is known that an interpretation in terms of active disks requires high mass accretion rates. These high accretion rates also lead to unreasonably small inclination angles for the disks. One might imagine that photo-evaporation of the disk due to UV radiation field of the central star could reduce the mass accretion rate in the inner disk (Richling \& Yorke 1997), thus allowing for discrepancies between the $\dot{M}$ derived from the infrared SED (which is most sensitive to the conditions in the outer disk) and that obtained from the hot continuum. However, this process requires the presence of a strong UV source that is not available for the T Tauri stars that we consider here. On the other hand, as suggested at the beginning of this section, the required disk mass is not as high as previously argued, because it turns out to be just a fraction of the stellar mass.

In the case where a smaller value for $\alpha$ is assumed, the required mass accretion rates, in line with the scaling relation of Sect. 3.3, are lower, but still closer to the higher values of Hartigan et al. (1995) than to the mutually consistent values of Valenti et al. (1993) and of Gullbring et al. (1998). In this case, however, the lower accretion luminosity is accompanied by an increasing importance of the irradiation term, quantified by the higher values of $a$, thus leading to a mixed case where both contributions (of irradiation from the central star and of internal heating of the disk) are important. Finally, note that the luminosity of the star $L_{\star}$ is smaller than usually estimated, mainly because we attribute part of the near-infrared luminosity to the disk, rather than to the star.

The interplay between standard models and the selfgravitating scenario might be more interesting for the "continuum" stars, for which the best fit accretion rates from the model studied in this paper turn out to be higher, but not dramatically, than what inferred from observations at shorter wavelengths (Gullbring et al. 2000). We should reiterate that, by our modeling procedure, we actually overestimate the mass accretion rate because the contribution of irradiation is neglected.

Figure 6 shows a significant discrepancy between the model curves and the data at short wavelengths, particularly for DR Tau and DG Tau. Here we are generally not concerned about the short wavelength data, because the optical and ultraviolet emission and its variability are well 

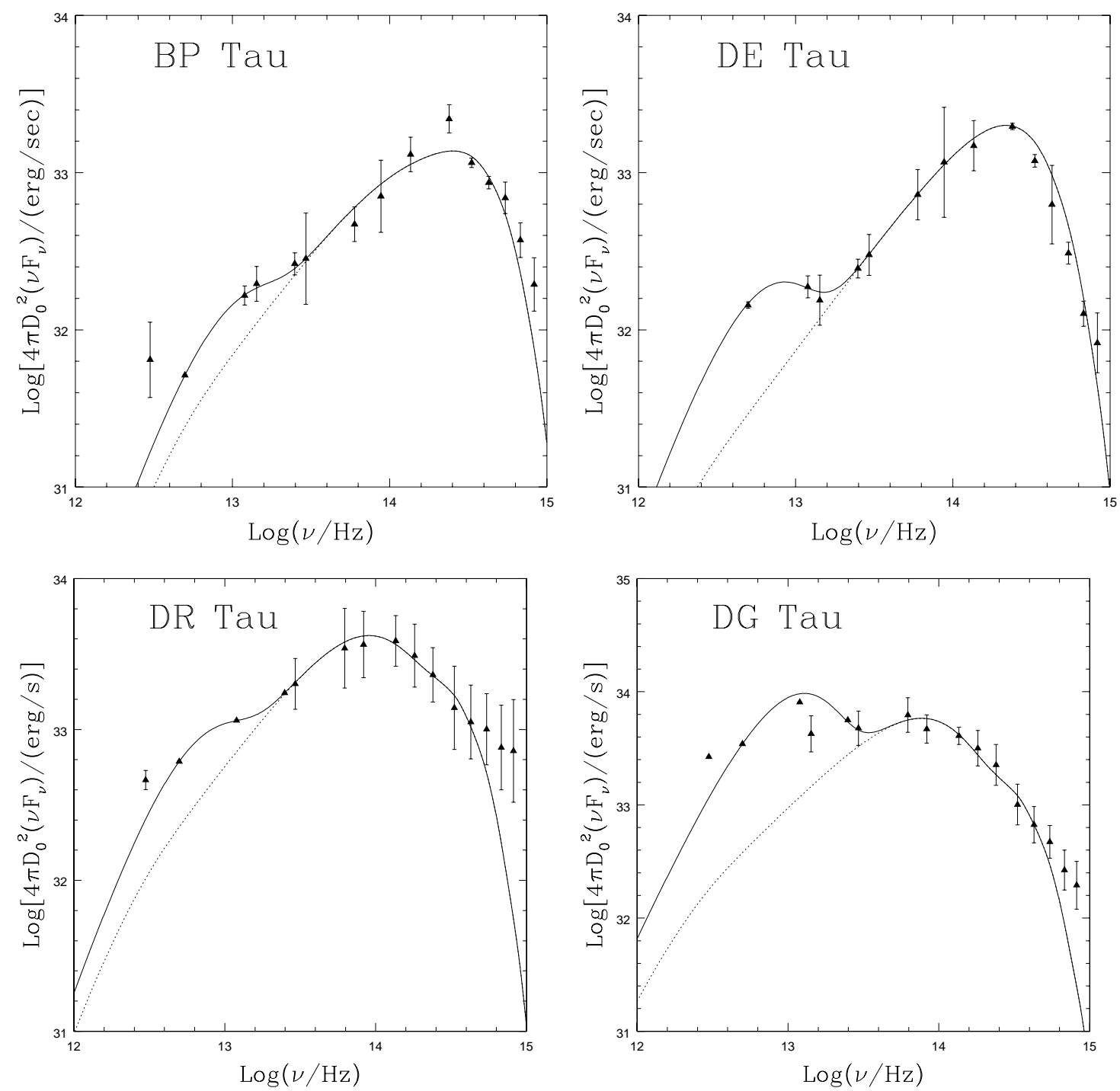

Fig. 6. Best fit to the spectral energy distributions of our sample stars (solid curve), compared to the spectra resulting from Keplerian disks with the same parameters $\left(\dot{M}, \cos \theta, x_{\mathrm{in}}, x_{\text {out }}\right.$; thin curves). For all cases the displayed fit refers to $x_{Q}=0.4$. The spectral data are from Kenyon \& Hartmann (1995) and Weaver \& Jones (1992).

interpreted in terms of magnetospheric accretion (e.g., see Kenyon et al. 1994).

\section{Conclusions}

In this paper we have described a model for selfgravitating accretion disks in the context of protostellar disks. This model might in principle be relevant for a number of cases, because the mass of the protostellar disk is likely to be high in the earlier phases of the accretion process. In addition, massive disks are sometimes observed in the context of intermediate mass star formation.

We have described the properties of the spectral energy distribution of these self-gravitating disks and their dependence on the various parameters involved. In addition, we have checked the parameter requirements for selfgravitating disks to fit the SEDs of some observed cases. In particular, we have considered the cases of FU Orionis objects and of some T Tauri stars.
For the FU Orionis objects we find that the infrared excess at wavelengths larger than $\lambda=10 \mu \mathrm{m}$ can be described in terms of the presence of a self-gravitating disk. Our models are otherwise consistent (for the implied mass accretion rate, for example) with previous models (see Kenyon \& Hartmann 1991), which, in contrast, attribute the far infrared excess to an infalling envelope.

We have also compared the spectral energy distribution of our models with the SEDs of some T Tauri stars, even though in general these should not be considered for a realistic application of our models, in order to check the requirements on an interpretation based on an active disk when self-gravity is taken into account. The required disk masses appear to be much lower than estimated previously. On the other hand, especially when the viscosity parameter is taken to be high, we do find unreasonably high required mass accretion rates. For typical $\mathrm{T}$ Tauri stars, our very simple models have an evident limitation, 

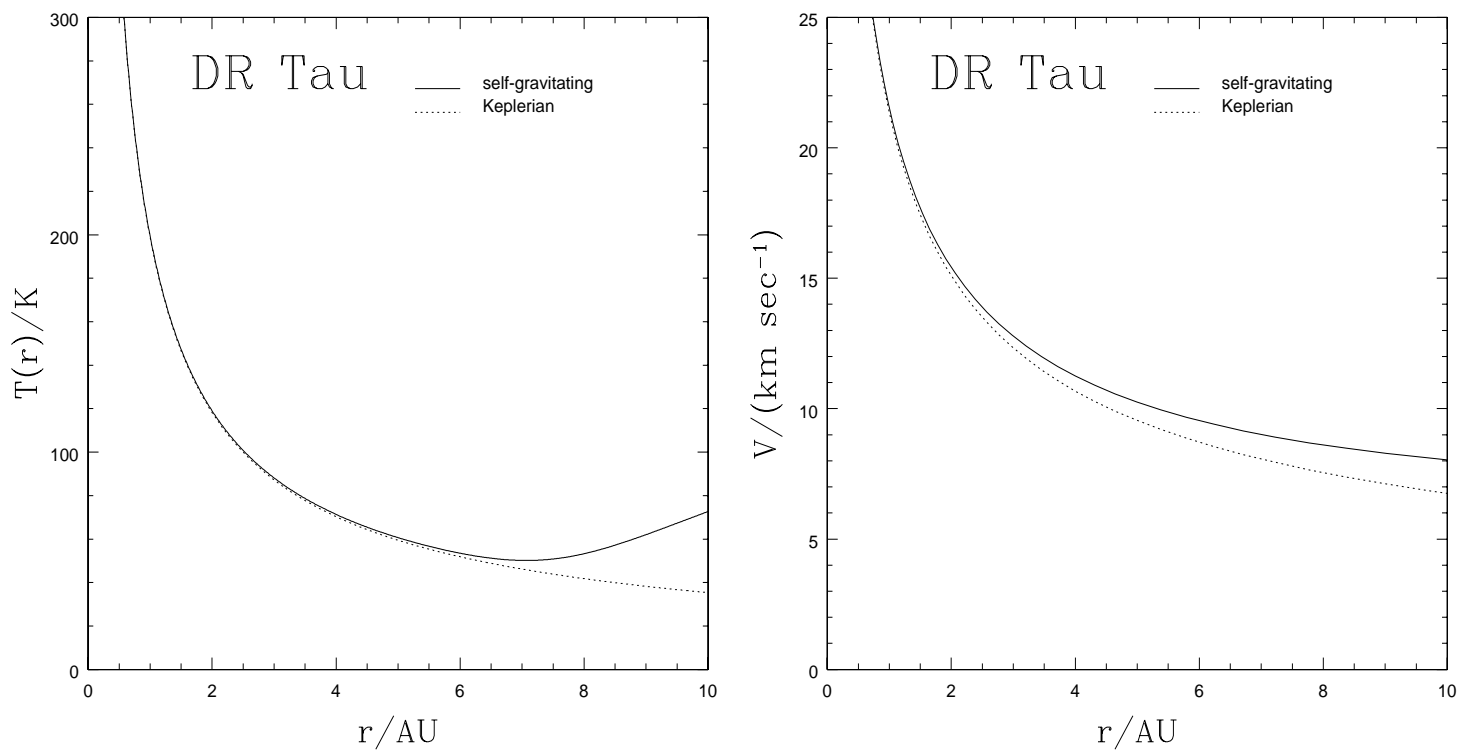

Fig. 7. Surface temperature profile and rotation curve for the best-fit model to DR Tau (with $x_{Q}=0.4$ ), compared with the corresponding Keplerian model.

Table A.1. Best-fit parameters for the T Tauri sample. The values in square brackets refer to different choices of the free parameter $x_{Q}$ ranging in the interval [0.4-0.9]. The values of $\alpha$ in parentheses are assumed (see discussion in Sect. 5).

\begin{tabular}{ccccccc}
\hline \hline & BP Tau & DE Tau & BP Tau & DE Tau & DR Tau & DG Tau \\
\hline $\log \left(\nu_{0} / H z\right)$ & {$[11.86-12.11]$} & {$[11.55-11.81]$} & {$[11.77-12.03]$} & {$[11.43-11.68]$} & {$[11.60-11.81]$} & {$[11.68-11.87]$} \\
$\log \left(L_{\star} /(\mathrm{erg} / \mathrm{s})\right)$ & 33.298 & 33.231 & 33.298 & 33.231 & 33.562 & 33.499 \\
$\log \left(L_{D} /(\mathrm{erg} / \mathrm{s})\right)$ & {$[29.80-30.14]$} & {$[29.42-29.77]$} & {$[29.67-30.02]$} & {$[29.26-29.60]$} & {$[30.34-30.63]$} & {$[30.62-30.89]$} \\
$x_{\text {in }}$ & {$[0.004-0.009]$} & {$\left[8 \times 10^{-4}-0.002\right]$} & {$[0.003-0.007]$} & {$\left[7 \times 10^{-4}-0.001\right]$} & {$[0.003-0.005]$} & {$[0.004-0.007]$} \\
$x_{\text {out }}$ & {$[0.60-1.32]$} & {$[0.75-1.63]$} & {$[0.60-1.06]$} & {$[0.56-1.25]$} & {$[0.54-1.09]$} & {$[0.81-1.56]$} \\
$\alpha$ & $\left(10^{-3}\right)$ & $\left(10^{-3}\right)$ & $\left(10^{-4}\right)$ & $\left(10^{-4}\right)$ & {$\left[2.2 \times 10^{-4}-0.8 \times 10^{-4}\right]$} & {$\left[4.7 \times 10^{-4}-1.8 \times 10^{-4}\right]$} \\
\hline
\end{tabular}

Table A.2. Derived parameters for the disks of the T Tauri sample stars.

\begin{tabular}{ccc|cc|cc}
\hline \hline \multicolumn{7}{c}{$\alpha=10^{-3}$} \\
\hline & BP Tau & DE Tau & BP Tau & DE Tau & DR Tau & DG Tau \\
\hline$\dot{M} /\left(10^{-6} M_{\odot} /\right.$ yr $)$ & {$[3-7]$} & {$[0.81-1.7]$} & {$[0.5-0.7]$} & {$[0.12-0.26]$} & {$[0.5-0.5]$} & {$[1-1]$} \\
$M_{\text {disk }} / M_{\odot}$ & {$[0.28-0.44]$} & {$[0.19-0.31]$} & {$[0.28-0.3]$} & {$[0.13-0.21]$} & {$[0.25-0.33]$} & {$[0.41-0.5]$} \\
$r_{\mathrm{s}} / \mathrm{AU}$ & {$[15-9]$} & {$[20-12]$} & {$[11-7]$} & {$[16-10]$} & {$[20-10]$} & {$[19-11]$} \\
$L_{\star} / L_{\odot}$ & 0.51 & 0.44 & 0.51 & 0.44 & 0.95 & 0.82 \\
$a / 10^{-1}$ & {$[0.06-0.02]$} & {$[0.3-0.2]$} & {$[0.4-0.1]$} & {$[3.5-1.5]$} & {$[1-1]$} & {$[0.4-0.4]$} \\
$\cos \theta$ & {$[0.03-0.02]$} & {$[0.14-0.08]$} & {$[0.12-0.07]$} & {$[0.53-0.31]$} & 1 & 1 \\
\hline
\end{tabular}

because we have not considered the important role of disk irradiation.

In general, we would like to see this paper as an interesting starting point for the inclusion of self-gravity related effects, even in the standard framework of the irradiated disk model.

Acknowledgements. We would like to thank L. Hartmann, for kindly providing us with the spectral data of FU Ori, and R. Cesaroni, A. Natta, and M. Romaniello, for interesting discussions. We would also like to thank an anonymous Referee, whose questions and suggestions have led to a substantial im- provement of the paper. This work has been partially supported by MURST of Italy.

\section{Appendix A: Fit parameters obtained from the SEDs of some $T$ Tauri systems}

In this appendix we report the detailed values of the fit parameters and of the physical quantities derived from the fit of some T Tauri stars presented in Sect. 5. Table A.1 shows the fit parameters (for BP Tau and DE Tau the parameters are shown for two adopted values of $\alpha$; see Sect. 5) and Table A.2 displays the derived parameters. 


\section{References}

Adams, F. C., Lada, C. J., \& Shu, F. H. 1988, ApJ, 326, 865

Adams, F. C., Ruden, S. P., \& Shu, F. H. 1989, ApJ, 347, 959

Armitage, P. J., Livio, M., \& Pringle, J. E. 2001, MNRAS, in press

Bardou, A., Heyvaerts, J., \& Duschl, W. J. 1998, A\&A, 337, 966

Beckwith, S. V. W. et al. 1990, AJ, 99, 924

Bell, K. R., et al. 1995, ApJ, 444, 376

Bertin, G. 1997, ApJ, 478, L71

Bertin, G., \& Lodato, G. 1999, A\&A, 350, 694 (BL99)

Bertin, G., \& Lodato, G. 2001, A\&A, 370, 342 (BL01)

Burrows, C. J., et al. 1996, ApJ, 473, 437

Calvet, N., et al. 1992, Rev. Mex. Aston. Astrofis., 24, 27

Calvet, N., et al. 1994, ApJ, 434, 330

Cardelli, J. A., Clayton, G. C., \& Mathis, J. S. 1989, ApJ, 345, 245

Cesaroni, R., et al. 1994, ApJ, 435, L137

Chiang, E. I., \& Goldreich, P. 1997, ApJ, 490, 368

Dullemond, C. P. 2000, A\&A, 361, L17

Duschl, W. J., Strittmatter, P. A., \& Biermann, P. L. 2000, A\&A, 357, 1123

Gullbring, E., Hartmann, L., Briceño, C., \& Calvet, N. 1998, ApJ, 492, 323

Gullbring, E., Calvet, N., Muzerolle, J., \& Hartmann, L. 2000, ApJ, 544, 927

Hartigan, P., Edwards, S., \& Ghandour, L. 1995, ApJ, 452, 736

Hartmann, L. 1998, Accretion processes in star formation (Cambridge University Press, Cambridge)

Hartmann, L., \& Kenyon, S. J. 1996, ARA\&A, 34, 207

Hohl, F. 1971, ApJ, 168, 343
Hohl, F. 1973, ApJ, 184, 353

Kenyon, S. J., \& Hartmann, L. 1987, ApJ, 323, 714

Kenyon, S. J., \& Hartmann, L. 1991, ApJ, 382, 664

Kenyon, S. J., \& Hartmann, L. 1995, ApJS, 101, 117

Kenyon, S. J., Hartmann, L., \& Hewett, R. 1988, ApJ, 325, 231

Kenyon, S. J., et al. 1994, AJ, 107, 2153

Laughlin, G., \& Bodenheimer, P. 1994, ApJ, 436, 335

Laughlin, G., \& Różyczka, M. 1996, ApJ, 456, 279

Lay, O. P., et al. 1994, ApJ, 434, L75

Lin, D. N. C., \& Pringle, J. E. 1987, MNRAS, 225, 607

Lin, D. N. C., \& Pringle, J. E. 1990, ApJ, 358, 515

Lynden-Bell, D., \& Pringle, J. E. 1974, MNRAS, 168, 603

Nelson, A. F., et al. 1998, ApJ, 502, 342

Nelson, A. F., et al. 2000, ApJ, 529, 357

Pickett, B. K., et al. 1998, ApJ, 504, 468

Pickett, B. K., et al. 2000, ApJ, 529, 1034

Popham, R., Kenyon, S. J., Hartmann, L., \& Narayan, R. 1996, ApJ, 473, 422

Richling, S., \& Yorke, H. W. 1997, A\&A, 327, 317

Ruden, S. P., \& Pollack, J. B. 1991, ApJ, 375, 740

Rydgren, A. E., Strom, S. E., \& Strom, K. M. 1976, ApJS, 30, 307

Shakura, N. J., \& Sunyaev, R. A. 1973, A\&A, 24, 337

Shu, F. H., Adams, F. C., \& Lizano, S. 1987, ARA\&A, 25, 23

Stapelfeldt, K. R., et al. 1998, ApJ, 502, L65

Testi, L., Natta, A., Sheperd, D. S., \& Wilner, D. J. 2001, preprint [astro-ph/0102473]

Valenti, J. A., Basri, G., \& Johns, C. M. 1993, AJ, 106, 2024

Weaver, W. B., \& Jones, G. 1992, ApJS, 78, 239

Wilner, D. J., et al. 2000, ApJ, 534, L101

Yorke, H. W., \& Bodenheimer, P. 1999, ApJ, 525, 330 